\title{
Unscented Kalman Filtering on Lie Groups
}

\author{
Martin BRosSARD*, Silvère BonNABEL* and Jean-Philippe CONDOMINES ${ }^{\dagger}$ \\ ${ }^{*}$ MINES ParisTech, PSL Research University, Centre for Robotics, 60 Boulevard Saint-Michel, 75006 Paris, France \\ †École Nationale de l'Aviation Civile - BP 54005, Toulouse Cedex 4, 31055, France
}

\begin{abstract}
In this paper, we first consider a simple Bayesian fusion problem in a matrix Lie group, and propose to tackle it using the unscented transform. The method is then leveraged to derive two simple alternative unscented Kalman filters on Lie groups, for both cases of noisy partial measurements of the state, and full state noisy measurements of the state on the group. The general method is applied to a robot localization problem, and results based on experimental data combined with extensive Monte-Carlo simulations at various noise levels illustrate the superiority of the approach over the standard UKF.
\end{abstract} tion.

Keywords-Lie groups, unscented Kalman filter, robot localiza-

\section{INTRODUCTION}

The problem of robot localization (and mapping) based on the fusion of various sensors has long been an important field. With the advent of probabilistic robotics methods within the field of mobile robotics, the role of accurate statistics for localization (and mapping) has been increasingly recognized. The Unscented Kalman Filter (UKF) introduced by roboticists $[1,2]$ has become prevalent as an alternative to the Extended Kalman Filter (EKF) that may improve estimation in various cases and spares the practitioner the computation of Jacobians. Besides, there has been various recent works that have evidenced the fact that the Lie group structure of the configuration space $S E(3)$ plays a prominent role in probabilistic robotics, see [3]-[9]. It has also been shown recently that a Lie group underlies the state space in SLAM, and that using a (Lie group) Invariant EKF [6], the consistency issues of the EKF-SLAM can be fixed [10].

In this paper we consider systems whose state is an unimodular matrix Lie group. Using exponential coordinates to define Gaussians on the group, we derive a Lie group version of the unscented Kalman filter. We consider two distinct problems: the problem of noisy partial measurement of the state that live in a vector space, and the problem of full state noisy measurement which is to be treated slightly differently, as the measurement then lives in the Lie group. For each type of measurement, the proposed UKF on Lie Groups consists of two different variants: one based on left multiplications, and the other based on right multiplications. It turns out the variant that works best depends on the problem at hand, and both should be tested when one has no clear insight on which should be the best.

\section{A. Links and Differences with Previous Literature}

The original UKF and its square-root implementation [11] have been extensively adapted and used for attitude estimation, that is, for filtering on the Lie group $S O(3)$. An UKF called USQUE [12] has become a standard filter for attitude estimation. The filter does not fully use the Lie group structure of the state space, notably the Lie exponential map, but rather takes advantage of the quaternion representation of $S O(3)$, which does not carry over to general Lie groups. Recent works have also advocated the use of particular probability densities on $S O(3)$, the so-called Fisher distributions, as an interesting alternative to the Lie exponential coordinates of [3], and have brought them to bear for unscented attitude estimation [13].

Another line of research uses the unscented transform on Lie groups and Lie exponential coordinates to derive uncertainty ellipsoids that are proved to contain with certainty the state, when faced with bounded sensor errors, see [14,15]. $[16,17]$ also introduced an Invariant UKF, as an UKF capable of taking into account the symmetries of the system's equations, for state spaces that are generally not Lie groups.

Regarding $S E(3)$ and more general Lie groups, one can essentially follow two slightly different routes to describe Gaussians in exponential coordinates. The first consists in directly defining a density on the group, that can be referred to as concentrated Gaussian, as in $[18,19]$. Those distributions were recently used for extended Kalman filtering on Lie groups by [20]. The second one consists in assuming the distribution to be normal in the Lie algebra, and then to map it to the group through the exponential map, as advocated by [5] for robotics applications, and at the heart of the Invariant EKF of [6,21]. In the present paper, we follow this second method. This yields a propagation step of the UKF that is identical to the method of [5] to compound poses. However, [5] does not treat Bayesian estimation on $S E(3)$, which is necessary to update the state of the UKF when given a novel measurement.

The very recent paper [22] also proposes an UKF on the Lie group $S E(3)$ for a navigation application. Beyond the fact it is only concerned with $S E(3)$ whereas the present paper deals with general Lie groups, the method is different since it uses concentrated Gaussians on Lie groups of [18]. As a result, the method can be related to the general unscented Kalman filtering on manifolds of [23], where the sigma points are mapped onto the manifold, and then the logarithm map is used to map them back in some tangent space where averages and covariances can be computed, whereas the sigma points in our method below live in a vector space, and are never mapped onto the group itself (regarding partial measurements). Last but not least, the method of [22] is based on left multiplications, notably on the left-invariant connection of $S E(3)$, whereas, as emphasized in the present paper, right multiplications allow defining an alternative UKF. This is important, as simulations of Section V-D indicate the right multiplications based UKF precisely suits best measurements in the body frame, such as those considered in [22]. 


\section{B. Paper's Organization and Contributions}

The paper is divided into six sections as follows. Section II contains mathematical preliminaries. Each of the following three sections constitutes a contribution of the present paper. In Section III, we design different Bayesian estimators on Lie groups based on the unscented transform for various kinds of measurement. The method is applied in Section IV to derive two UKF on Lie groups. Section V considers 2D robot localization problem that uses experimental data, and illustrates the superiority of the proposed UKF over the standard UKF and the Invariant EKF.

\section{Mathematical Preliminaries}

\section{A. Lie Groups}

In this section we recall the definitions and basic properties of unimodular matrix Lie groups, Lie algebra and random variables on Lie groups. A matrix Lie group $G \subset \mathbb{R}^{N \times N}$ is a set of square invertible matrices that is a group, i.e., the following properties

$$
\mathbf{I} \in G ; \forall \chi \in G, \chi^{-1} \in G ; \forall \chi_{1}, \chi_{2} \in G, \chi_{1} \chi_{2} \in G
$$

hold. Locally about the identity matrix $\mathbf{I}$, the group $G$ can be identified with an Euclidean space $\mathbb{R}^{q}$ using the matrix exponential map $\exp _{m}($.$) , where q=\operatorname{dim} G$. Indeed, to any $\boldsymbol{\xi} \in \mathbb{R}^{q}$ one can associate a matrix $\boldsymbol{\xi}^{\wedge}$ of the tangent space of $G$ at I, called the Lie algebra $\mathfrak{g}$. We then define the exponential map exp : $\mathbb{R}^{q} \rightarrow G$ for Lie groups as

$$
\exp (\boldsymbol{\xi})=\exp _{m}\left(\boldsymbol{\xi}^{\wedge}\right),
$$

Locally, it is a bijection, and one can define the Lie logarithm map $\log : G \rightarrow \mathbb{R}^{q}$ as the exponential inverse, leading to

$$
\log (\exp (\boldsymbol{\xi}))=\boldsymbol{\xi} .
$$

\section{B. Uncertainties on Lie Groups}

To define random variables on Lie groups, we cannot apply the usual approach of additive noise for $\chi_{1}, \chi_{2} \in G$ as $G$ is not a vector space, i.e., generally $\chi_{1}+\chi_{2} \notin G$ does not hold. In contrast, we adopt the framework of [5], see also [6,21], which is slightly different from the pioneering approach of [3,24]. Indeed, we define the probability distribution $\chi \sim \mathcal{N}_{\mathrm{L}}(\bar{\chi}, \mathbf{P})$ for the random variable $\chi \in G$ as

$$
\chi=\bar{\chi} \exp (\boldsymbol{\xi}), \boldsymbol{\xi} \sim \mathcal{N}(\mathbf{0}, \mathbf{P}),
$$

where $\mathcal{N}(.,$.$) is the classical Gaussian distribution in \mathrm{Eu}$ clidean space and $\mathbf{P} \in \mathbb{R}^{q \times q}$ is a covariance matrix. In the sequel, we will refer to (4) as the left-equivariant Gaussian distribution on $G$, due to its compatibility with left multiplications. In (4), the noise-free quantity $\bar{\chi}$ is viewed as the mean, and the dispersion arises through left multiplication with the exponential of a Gaussian random variable. Similarly, the distribution $\chi \sim \mathcal{N}_{\mathrm{R}}(\bar{\chi}, \mathbf{P})$ can be defined through right multiplication as

$$
\chi=\exp (\boldsymbol{\xi}) \bar{\chi}, \boldsymbol{\xi} \sim \mathcal{N}(\mathbf{0}, \mathbf{P}) .
$$

We stress that we have defined these probability density functions directly in the vector space $\mathbb{R}^{q}$ such that both $\mathcal{N}_{\mathrm{L}}(.,$. and $\mathcal{N}_{\mathrm{R}}(.,$.$) are not Gaussian distributions.$

\section{BAYESIAN ESTIMATION ON LIE GROUPS USING THE UNSCENTED TRANSFORM}

Consider a random variable $\chi \in G$ with prior probability distribution $p(\boldsymbol{\chi})$. Suppose we obtain some additional information about $\chi$ through a measurement $\mathbf{y}$. The goal of Bayesian estimation is to compute an estimate of the posterior distribution $p(\boldsymbol{\chi} \mid \mathbf{y})$. In this section, we derive various algorithms for various types of measurements.

\section{A. Partial Measurement with Left-equivariant Prior Uncer- tainty (4)}

Consider a generic vector measurement of the form

$$
\mathbf{y}=h(\boldsymbol{\chi})+\mathbf{v},
$$

where $h():. G \rightarrow \mathbb{R}^{k}$ represents the observation function and $\mathbf{v} \sim \mathcal{N}(\overline{\mathbf{v}}, \mathbf{R})$ is a Gaussian random noise in $\mathbb{R}^{k}$ with known characteristics. The problem of Bayesian estimation we consider is as follows:

1) consider the prior distribution follows (4), i.e.,

$$
p(\chi) \sim \mathcal{N}_{\mathrm{L}}(\bar{\chi}, \mathbf{P}),
$$

with known parameters $\bar{\chi}$ and $\mathbf{P}$;

2) assume one measurement $\mathbf{y}$ given by (6) is available;

3) approximate the posterior distribution as

$$
p(\boldsymbol{\chi} \mid \mathbf{y}) \approx \mathcal{N}_{\mathrm{L}}\left(\overline{\boldsymbol{\chi}}^{+}, \mathbf{P}^{+}\right),
$$

and compute the estimates of both posterior parameters $\bar{\chi}^{+}$and $\mathbf{P}^{+}$.

To attack this problem, one can resort the unscented transform of [1]. First, and contrarily to [22], we use the fact that the problem is amenable to nonlinear filtering in a vector space, as we have $\boldsymbol{\xi} \sim \mathcal{N}(\mathbf{0}, \mathbf{P})$ with nonlinear measurement (6). The UKF thus allows us to approximate the posterior $p(\boldsymbol{\xi} \mid \mathbf{y})$ for $\boldsymbol{\xi}$ as follows: we compute a finite number of samples $\boldsymbol{\alpha}_{j}^{\top}=$ $\left[\boldsymbol{\xi}_{j}^{\top} \mathbf{v}_{j}^{\top}\right], j=0, \ldots, 2 l$, with $l=q+k$, and then pass each of these so-called sigma points through the measurement function

$$
\mathbf{y}_{j}=h\left(\bar{\chi} \exp \left(\boldsymbol{\xi}_{j}\right)\right)+\mathbf{v}_{j}, j=0, \ldots, 2 l,
$$

and we then compute successively the measurement mean $\overline{\mathbf{y}}$, the measurement covariance $\mathbf{P}_{\mathbf{y y}}$ and the cross-covariance $\mathbf{P}_{\boldsymbol{\alpha} \mathbf{y}}$. We thus approximate the posterior for $\boldsymbol{\xi}$ in $\mathbb{R}^{q}$ as

$$
\begin{aligned}
& p(\boldsymbol{\xi} \mid \mathbf{y}) \sim \mathcal{N}\left(\overline{\boldsymbol{\xi}}, \mathbf{P}^{+}\right), \text {where } \\
& {\left[\begin{array}{l}
\overline{\boldsymbol{\xi}} \\
*
\end{array}\right]=\mathbf{P}_{\boldsymbol{\alpha y}} \mathbf{P}_{\mathbf{y y}}^{-1}(\mathbf{y}-\overline{\mathbf{y}}) \text { and }} \\
& \mathbf{P}^{+}=\mathbf{P}-\mathbf{P}_{\boldsymbol{\alpha y}}\left(\mathbf{P}_{\boldsymbol{\alpha} \mathbf{y}} \mathbf{P}_{\mathbf{y y}}^{-1}\right)^{\top} .
\end{aligned}
$$

The unscented approximation to the posterior $p(\boldsymbol{\xi} \mid \mathbf{y})$ is thus the distribution of a Gaussian $\overline{\boldsymbol{\xi}}+\boldsymbol{\xi}^{+}$with $\boldsymbol{\xi}^{+} \sim \mathcal{N}\left(\mathbf{0}, \mathbf{P}^{+}\right)$. Back to the Lie group, this means we approximate the posterior distribution through the variable $\bar{\chi} \exp \left(\overline{\boldsymbol{\xi}}+\boldsymbol{\xi}^{+}\right)$. The BakerCampbell-Hausdorff $(\mathrm{BCH})$ formula provides a simple (firstorder) approximation as $\exp \left(\overline{\boldsymbol{\xi}}+\boldsymbol{\xi}^{+}\right) \approx \exp (\overline{\boldsymbol{\xi}}) \exp \left(\boldsymbol{\xi}^{+}\right)$. This readily yields an approximate $\bar{\chi}^{+} \exp \left(\boldsymbol{\xi}^{+}\right)$to the posterior of the form (4), with

$$
\bar{\chi}^{+}=\bar{\chi} \exp (\overline{\boldsymbol{\xi}}), \boldsymbol{\xi}^{+} \sim \mathcal{N}\left(\mathbf{0}, \mathbf{P}^{+}\right),
$$


that concludes the Bayesian estimation, which is summarized in Algorithm $1^{1}$.

\section{B. Partial Measurement with Right-equivariant Prior Uncer- tainty (5)}

In the latter subsection, we considered that both the prior and posterior follow the distribution of the form (4). If we assume the prior has the form (5), then it is natural to assume that the posterior is also of the form (5). In this case, we substitute the computed measurements $\mathbf{y}_{j}, j=0, \ldots, 2 l$ in (9) as

$$
\mathbf{y}_{j}=h\left(\exp \left(\boldsymbol{\xi}_{j}\right) \bar{\chi}\right)+\mathbf{v}_{j}
$$

and the posterior mean (13) as

$$
\bar{\chi}^{+}=\exp (\overline{\boldsymbol{\xi}}) \overline{\boldsymbol{\chi}}
$$

The difference between both methods may prove nonnegligible as will be illustrated in Section V-D.

\section{Full State Measurement: Bayesian Pose Fusion}

Assume we have a prior of the form $p(\boldsymbol{\chi}) \sim \mathcal{N}_{\mathrm{L}}(\overline{\boldsymbol{\chi}}, \mathbf{P})$ and a measurement of the type

$$
\mathbf{Y}=\chi \mathbf{V}
$$

where $\chi$ is the true state and $\mathbf{V}$ a noise of the form

$$
\mathbf{V}=\exp (\mathbf{v}), \mathbf{v} \sim \mathcal{N}(\mathbf{0}, \mathbf{R}) .
$$

This kind of measurement happens for instance in $S E(3)$, when an algorithm is used to compute a pose directly from perception sensors such as, stereo images, or scan matching algorithms with point clouds, and when a covariance can be associated to the computed pose, see e.g., [26].

1) Proposed Method: in this case, we need to approximate the posterior $p(\chi \mid \mathbf{Y}) \sim \mathcal{N}_{\mathrm{L}}\left(\bar{\chi}^{+}, \mathbf{P}^{+}\right)$, which appears to be nontrivial. Indeed, in (16), $\mathbf{Y}$ is a matrix measurement, and this matrix belongs to $G$ which is not a vector space, so both the definition and the computation of the covariance of $\mathbf{Y}$ is not straightforward. For such measurement, we propose using the logarithm map to obtain

$$
\begin{aligned}
\mathbf{y} & =\log \left(\overline{\boldsymbol{\chi}}^{-1} \mathbf{Y}\right)=\log \left(\overline{\boldsymbol{\chi}}^{-1} \boldsymbol{\chi} \mathbf{V}\right), \\
& =\log (\exp (\boldsymbol{\xi}) \mathbf{V}) .
\end{aligned}
$$

Thanks to this transformation, the computed transformed output $\mathbf{y}$ belongs to vector space $\mathbb{R}^{q}$, and we can then apply the proposed Bayesian estimation of Section III-A, with

$$
\mathbf{y}_{j}=\log \left(\exp \left(\boldsymbol{\xi}_{j}\right) \exp \left(\mathbf{v}_{j}\right)\right), j=0, \ldots, 2 l,
$$

to obtain the posterior $p(\boldsymbol{\chi} \mid \mathbf{Y}) \sim \mathcal{N}_{\mathrm{L}}\left(\overline{\boldsymbol{\chi}}^{+}, \mathbf{P}^{+}\right)$.

2) Fusion with Right-equivariant Prior Uncertainty (5): assume that we have a prior of the form (5), or by symmetry (inverting all quantities), that we have a left prior of the form (4) but with an output of the form $\mathbf{Y}=\mathbf{V} \chi$. We then

\footnotetext{
${ }^{1}$ According to $[1,25]$, we set unscented transform parameters to $\kappa=0$ and $\beta=2$. $\alpha$ keeps a free-parameter chosen by the practitioner, which must be small ( $\alpha=10^{-3}$ in our applications). The operator $\operatorname{diag}($.$) put in a$ block diagonal matrix its arguments in Step 3, the square-root matrix in step 4 is obtained from Cholewski decomposition and $\operatorname{col}(\mathbf{P})_{j}$ represents the $j$-th column of $\mathbf{P}$.
}

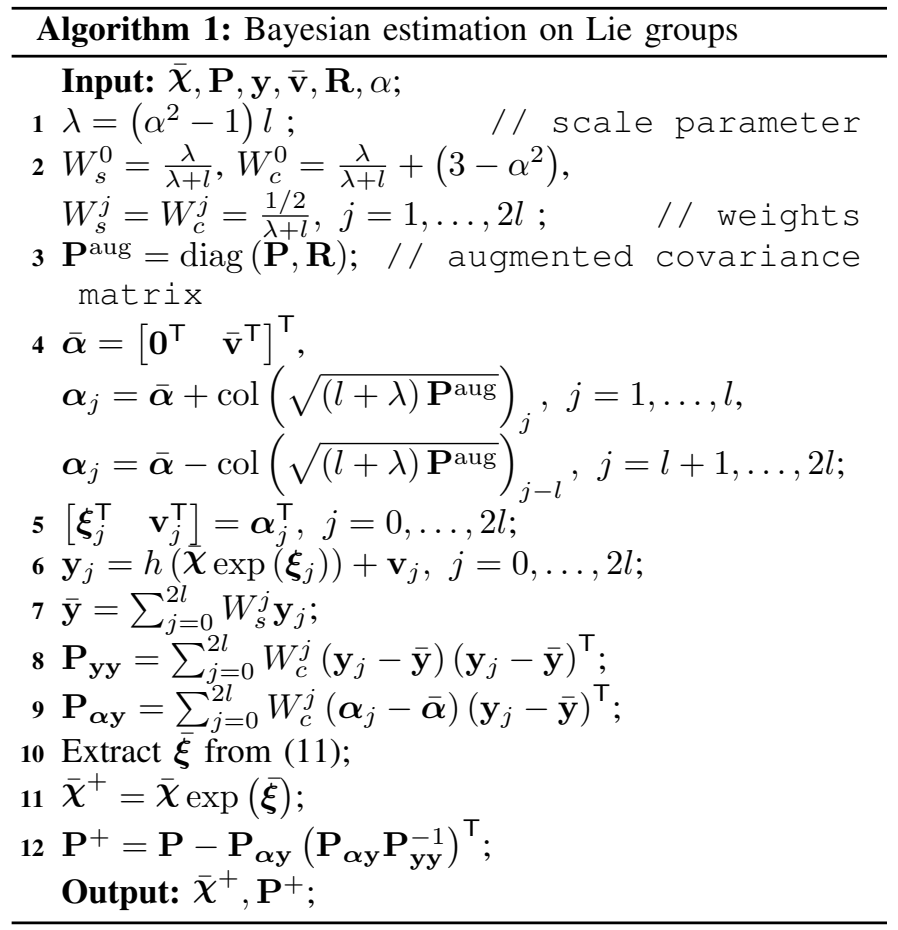

propose to write $\mathbf{Y}=\mathbf{V} \overline{\boldsymbol{\chi}} \exp (\boldsymbol{\xi})=\bar{\chi} \overline{\boldsymbol{\chi}}^{-1} \mathbf{V} \overline{\boldsymbol{\chi}} \exp (\boldsymbol{\xi})=$ $\overline{\boldsymbol{\chi}} \overline{\boldsymbol{\chi}}^{-1} \exp (\mathbf{v}) \overline{\boldsymbol{\chi}} \exp (\boldsymbol{\xi})=\overline{\boldsymbol{\chi}} \exp \left(\mathrm{Ad}_{\overline{\boldsymbol{\chi}}}^{-1} \mathbf{v}\right) \exp (\boldsymbol{\xi})$ where $\mathrm{Ad}$ is the adjoint operator of the group. We can thus compute the modified output

$$
\mathbf{y}=\log \left(\overline{\boldsymbol{\chi}}^{-1} \mathbf{Y}\right)=\log \left(\exp \left(\operatorname{Ad}_{\overline{\boldsymbol{\chi}}^{-1} \mathbf{v}}\right) \exp (\boldsymbol{\xi})\right) .
$$

Thanks to this transformation, we recover a measurement similar to (19), but with noise having covariance matrix $\operatorname{Ad}_{\bar{\chi}^{-1}} \mathbf{R}\left(\operatorname{Ad}_{\bar{\chi}^{-1}}\right)^{\top}$. The method above can then be readily applied.

3) Rationale for the Transformation of the Output: consider output (16). One could argue that $\mathbf{y}=\log (\mathbf{Y})$ is already a vector, and a function of $\boldsymbol{\xi}$ so that an UKF estimate can readily be built along the lines of Section III-A. However, when $\bar{\chi}$ is far from the identity, a large distortion is induced by the logarithm map. But $\boldsymbol{\xi}$ and $\mathbf{v}$ being moderate zerocentered noises in applications, we have using $\mathrm{BCH}$ formula $\log (\exp (\boldsymbol{\xi}) \mathbf{V})=\boldsymbol{\xi}+\mathbf{v}+o(\|\boldsymbol{\xi}\|,\|\mathbf{v}\|)$. Thus up to first order terms the transformed output $\mathbf{y}$ of (19) is a true Gaussian, and the posterior output by the UKF will be very close to the true one, no matter $\bar{\chi}$, whereas $\log (\mathbf{Y})$ can be dramatically nonGaussian, preventing the UKF estimate from being accurate.

4) Links with the Pose Fusion Method of [5]: [5] proposes an algorithm to fuse various measurements of the form (16). With two measurements only, it boils down to estimating an unknown group element $\bar{\chi}$ from measurements $\mathbf{Y}_{1}=\bar{\chi} \exp \left(\mathbf{v}_{1}\right)$ and $\mathbf{Y}_{2}=\bar{\chi} \exp \left(\mathbf{v}_{2}\right)$, where $\mathbf{v}_{1}$ and $\mathbf{v}_{2}$ are centered Gaussians in $\mathbb{R}^{q}$ with known covariance matrices. In the Euclidean case, the corresponding problem is to estimate some quantity $\boldsymbol{\mu}$ from two noisy measurements $\mathbf{y}_{1}=\boldsymbol{\mu}+\mathbf{n}_{1}$ and $\mathbf{y}_{2}=\boldsymbol{\mu}+\mathbf{n}_{2}$ where $\mathbf{n}_{1}$ and $\mathbf{n}_{2}$ are centered Gaussian noises with known covariance matrices. This is a classical estimation problem, that is optimally handled through 
least squares, and does not imply the Kalman update equation (Gaussian conditioning). The problem considered in the present section is quite different, since it deals with Bayesian fusion of poses, which implies conditional probabilities. In our case $\bar{\chi}$ is known, and we want to refine our statistics on the random quantity $\bar{\chi} \exp (\boldsymbol{\xi})$, given that we have measured, e.g., $\mathbf{Y}=\bar{\chi} \exp (\boldsymbol{\xi}) \exp (\mathbf{v})$.

\section{Application to Unscented Kalman Filtering ON LIE GROUPS}

\section{A. System Model}

In the rest of this paper we consider a discrete dynamic system of the form

$$
\chi_{n}=\chi_{n-1} \exp \left(\boldsymbol{\omega}_{n}+\mathbf{w}_{n}\right),
$$

where the state $\boldsymbol{\chi}_{n}$ lives in $G, \boldsymbol{\omega}_{n}$ is a known input variable and $\mathbf{w}_{n} \sim \mathcal{N}\left(\mathbf{0}, \mathbf{Q}_{n}\right)$ is a white Gaussian noise. We design two different filters for generic discrete measurements of the form

$$
\mathbf{y}_{n}=h\left(\boldsymbol{\chi}_{n}\right)+\mathbf{v}_{n}
$$

where $\mathbf{v}_{n} \sim \mathcal{N}\left(\overline{\mathbf{v}}_{n}, \mathbf{R}_{n}\right)$ is a white Gaussian noise with known characteristics, and also for measurements of the form

$$
\mathbf{Y}_{n}=\chi_{n} \exp \left(\mathbf{v}_{n}\right)
$$

where $\mathbf{v}_{n} \sim \mathcal{N}\left(\overline{\mathbf{v}}_{n}, \mathbf{R}_{n}\right)$.

\section{B. Unscented Kalman Filter on Lie Groups with Left- equivariant Uncertainties (4)}

For the model (22) with measurements given by (23), we can model the state as $\chi_{n} \sim \mathcal{N}_{\mathrm{L}}\left(\overline{\boldsymbol{\chi}}_{n}, \mathbf{P}_{n}\right)$, that is, using the left-equivariant formulation (4) of the uncertainties. The goal is to compute both estimates of $\bar{\chi}_{n}$ and $\mathbf{P}_{n}$ at each time. The proposed Left-Unscented Kalman Filter on Lie Groups (LeftUKF-LG) acts in two steps: propagation and update. We now develop these two steps.

1) Propagation: starting from the prior distribution

$$
p\left(\boldsymbol{\chi}_{n-1}\right) \sim \mathcal{N}_{\mathrm{L}}\left(\overline{\boldsymbol{\chi}}_{n-1}, \mathbf{P}_{n-1}\right),
$$

with $\bar{\chi}_{n-1}$ and $\mathbf{P}_{n-1}$ known, we seek to approximate the propagated state distribution

$$
p\left(\boldsymbol{\chi}_{n} \mid \chi_{n-1}\right) \sim \mathcal{N}_{\mathrm{L}}\left(\overline{\boldsymbol{\chi}}_{n}, \mathbf{P}_{n}\right),
$$

i.e., we search to compute both $\bar{\chi}_{n}$ and $\mathbf{P}_{n}$.

This step can be viewed as a straightforward application of the method of [5] to compound poses using the unscented transform. The state mean estimate is propagated using the unnoisy state model, leading to

$$
\bar{\chi}_{n}=\bar{\chi}_{n-1} \boldsymbol{\Omega}_{n}
$$

with $\boldsymbol{\Omega}_{n}=\exp \left(\boldsymbol{\omega}_{n}\right)$, According to [5] this approximation of the mean is valid up to the second order, so there is no need to compute it through the unscented transform (the calculations of [5] deal with $S E(3)$, but we have checked they carry over to the general Lie group case). We then resort to the unscented transform to determine the covariance $\mathbf{P}_{n}$ by first approximating the joint Gaussian variable using a finite

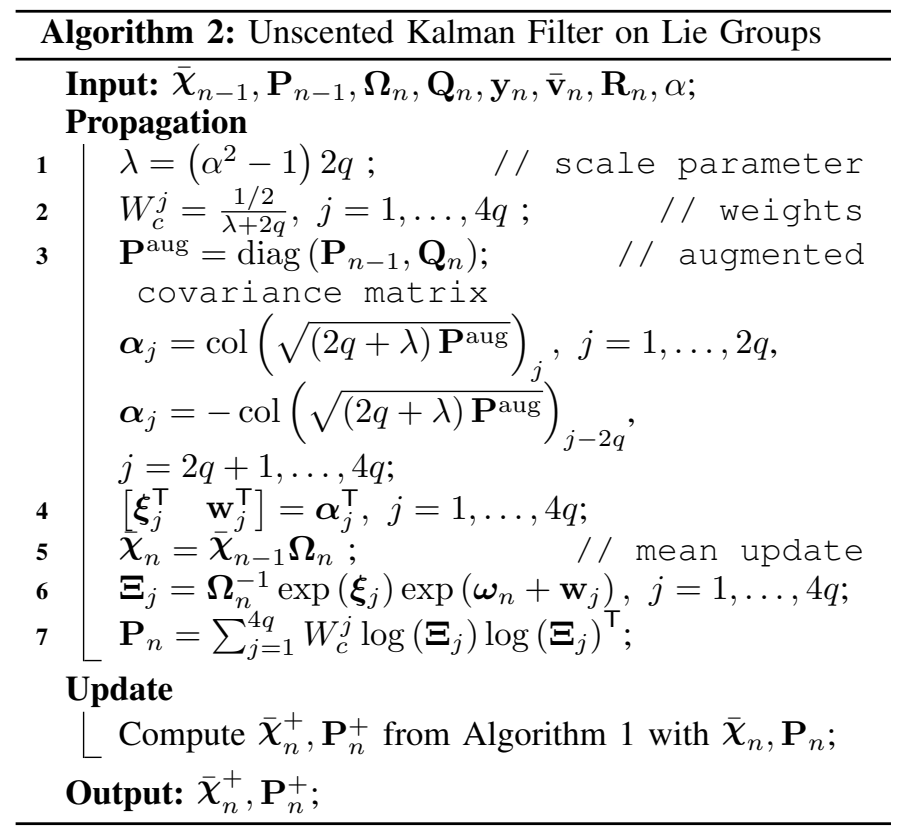

number of samples $\boldsymbol{\xi}_{j}, \mathbf{w}_{j}, j=1, \ldots, 4 q$. To propagate theses samples, we inject (4) in (22) as

$$
\overline{\boldsymbol{\chi}}_{n} \exp \left(\boldsymbol{\xi}_{n}\right)=\overline{\boldsymbol{\chi}}_{n-1} \exp \left(\boldsymbol{\xi}_{n-1}\right) \exp \left(\boldsymbol{\omega}_{n}+\mathbf{w}_{n}\right),
$$

which simplifies as follows

$$
\begin{aligned}
& \exp \left(\boldsymbol{\xi}_{n}\right)=\overline{\boldsymbol{\chi}}_{n}^{-1} \overline{\boldsymbol{\chi}}_{n-1} \exp \left(\boldsymbol{\xi}_{n-1}\right) \exp \left(\boldsymbol{\omega}_{n}+\mathbf{w}_{n}\right), \\
& \exp \left(\boldsymbol{\xi}_{n}\right)=\boldsymbol{\Omega}_{n}^{-1} \exp \left(\boldsymbol{\xi}_{n-1}\right) \exp \left(\boldsymbol{\omega}_{n}+\mathbf{w}_{n}\right),
\end{aligned}
$$

by use of (27). Algorithm 2 summarizes this propagation step.

2) Update: this step consists in incorporating the information coming from the measurement. As concerns measurements $\mathbf{y}_{n}$ of the form (23), it boils down to a Bayesian estimation problem, where we search to approximate the posterior probability distribution as

$$
p\left(\boldsymbol{\chi}_{n} \mid \mathbf{y}_{n}, \mathbf{y}_{n-1}, \ldots, \mathbf{y}_{1}\right) \sim \mathcal{N}_{\mathrm{L}}\left(\overline{\boldsymbol{\chi}}_{n}^{+}, \mathbf{P}_{n}^{+}\right)
$$

with available prior

$$
p\left(\boldsymbol{\chi}_{n} \mid \mathbf{y}_{n-1}, \ldots, \mathbf{y}_{1}\right) \sim \mathcal{N}_{\mathrm{L}}\left(\overline{\boldsymbol{\chi}}_{n}, \mathbf{P}_{n}\right) .
$$

We apply for the update estimates directly Bayesian estimation as developed in Section III-A, yielding both $\overline{\boldsymbol{\chi}}_{n}^{+}$and $\mathbf{P}_{n}^{+}$.

To update the filter with a measurement of the type (24), it suffices to apply in a similar manner the method of Section III-C.

\section{Unscented Kalman Filter on Lie Groups with Right- equivariant Uncertainties (5)}

For the model (22) with measurements given by (23), we can model the state as $\chi_{n} \sim \mathcal{N}_{\mathrm{R}}\left(\bar{\chi}_{n}, \mathbf{P}_{n}\right)$, that is, using the right-equivariant formulation (5) of the uncertainties. The filter is defined in an analogous way to the previous Section IV-B. The resulting filter is yet different, and we call it RightUnscented Kalman Filter on Lie Groups (Right-UKF-LG). 


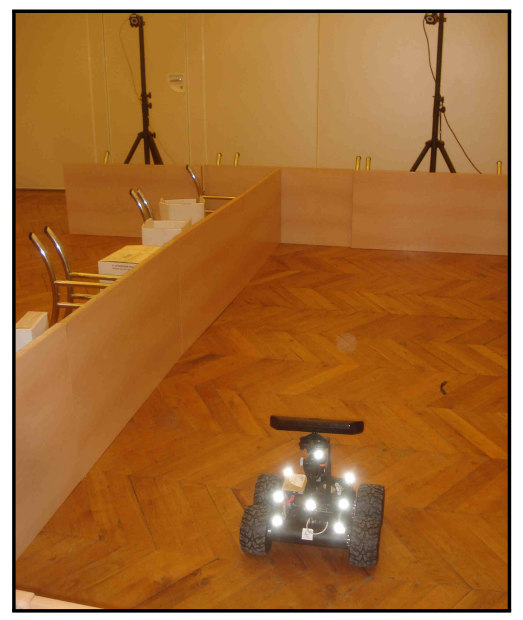

Fig. 1: Testing arena with Wifibot robot in the foreground of the picture. We can also see two of the seven Optitrack cameras in the background.

Section V-D evidences that large discrepancies in performance between the Left and Right UKF-LGs can occur, so that both variants should generally be tested when facing a novel estimation problem on Lie groups.

\section{RESUlTS FOR EXPERIMENTAL DATA}

To demonstrate the performances of the proposed method, we first apply the proposed Left-UKF-LG to the following nonlinear standard model of the two-dimensional differential drive car [27], and compare it to various algorithms of the literature. The velocity $u_{n} \in \mathbb{R}$ is given by an odometer and we measure the angular velocity $\omega_{n} \in \mathbb{R}$, e.g., by a differential odometer or a gyrometer. Taking into account the possible noise in the measurements, we get the discrete model

$$
\begin{aligned}
& \theta_{n}=\theta_{n-1}+\omega_{n}+w_{n}^{\theta}, \\
& x_{n}^{1}=x_{n-1}^{1}+\cos \left(\theta_{n}\right)\left(u_{n}+w_{n}^{1}\right)-\sin \left(\theta_{n}\right) w_{n}^{\mathrm{tr}}, \\
& x_{n}^{2}=x_{n-1}^{2}+\sin \left(\theta_{n}\right)\left(u_{n}+w_{n}^{1}\right)+\cos \left(\theta_{n}\right) w_{n}^{\mathrm{tr}},
\end{aligned}
$$

where $w_{n}^{\theta}, w_{n}^{1}$ and $w_{t}^{\mathrm{tr}}$ represent, respectively, the differential odometry error, the longitudinal odometry error and the transversal shift, whose introduction is thus physically motivated. We assume the vehicle obtains some noisy measurements of its position of the form

$$
\tilde{\mathbf{y}}_{n}=\mathbf{x}_{n}+\tilde{\mathbf{v}}_{n}, \quad \tilde{\mathbf{v}}_{n} \sim \mathcal{N}\left(\mathbf{0},\left[\begin{array}{cc}
\sigma^{2} & 0 \\
0 & \sigma^{2}
\end{array}\right]\right),
$$

which typically models a GPS position in an outdoor application. The system can be embedded in the matrix Lie group $G=S E(2)$ as explained in Appendix A, using $\chi_{n}$ as the state, and

$$
\boldsymbol{\omega}_{n}=\left[\begin{array}{c}
\omega_{n} \\
u_{n} \\
0
\end{array}\right] \text { and } \mathbf{w}_{n}=\left[\begin{array}{c}
w_{n}^{\theta} \\
w_{n}^{1} \\
w_{n}^{\mathrm{tr}}
\end{array}\right] .
$$

The equation governing the noisy system evolution then becomes

$$
\boldsymbol{\chi}_{n}=\boldsymbol{\chi}_{n-1} \exp \left(\boldsymbol{\omega}_{n}+\mathbf{w}_{n}\right)
$$

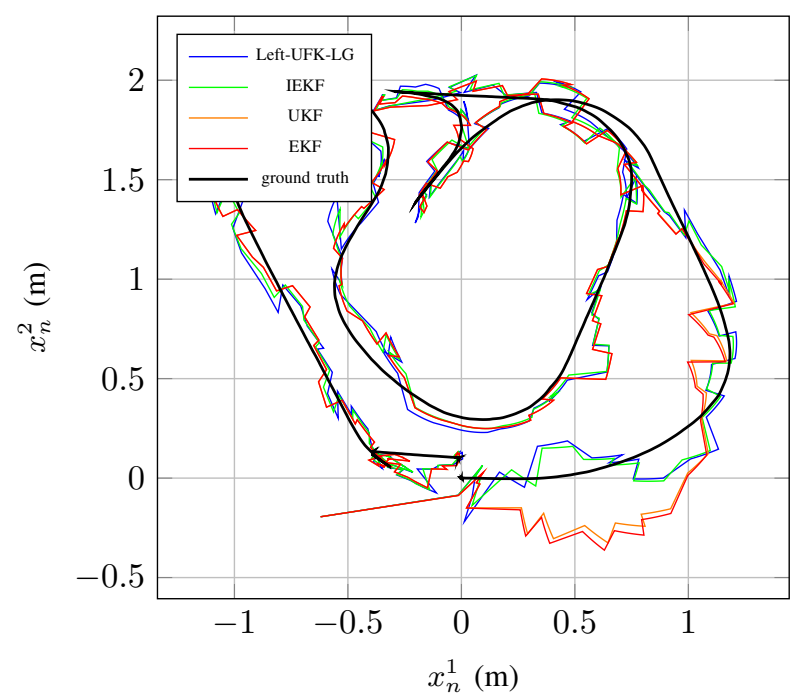

Fig. 2: Ground truth trajectory starting from the position $(0,0)$ and estimated trajectories with wrong initial positions. The noise is moderate to high $\left(\sigma^{2}=10^{-2} \mathrm{~m}^{2}\right)$. The standard UKF and EKF encounter serious difficulties at the beginning due to initial dispersion encoded in $\mathbf{P}_{0}$.

and the observations have the equivalent form

$$
\mathbf{y}_{n}=\left[\begin{array}{c}
\mathbf{x}_{n}+\tilde{\mathbf{v}}_{n} \\
1
\end{array}\right]=\underbrace{\boldsymbol{\chi}_{n}\left[\begin{array}{l}
\mathbf{0} \\
1
\end{array}\right]}_{h\left(\boldsymbol{\chi}_{n}\right)}+\underbrace{\left[\begin{array}{c}
\tilde{\mathbf{v}}_{n} \\
0
\end{array}\right]}_{\mathbf{v}_{n}} .
$$

\section{A. Experimental Setting}

We then compare the various filters for the model described in Section V on real data obtained in an experiment conducted at the Centre for Robotics, MINES ParisTech. We used a socalled Wifibot, which is a small wheeled robot equipped with independent odometers on the left and right wheels, see Fig. 1. A set of seven highly precise cameras, the OptiTrack motion capture system, provide the reference trajectory (ground truth) $\theta_{n}$ and $\mathbf{x}_{n}$ with sub-millimeter precision at a rate of $120 \mathrm{~Hz}$. We display the experimental trajectory in Fig. 2, corresponding to a random motion of 45 seconds. From this trajectory, we can determine the odometer noise characteristics to parametrize correctly the covariance matrix $\mathbf{Q}_{n}$.

The four filters to be compared are:

1) the standard EKF using the model original equations (33) with state variables $\mathbf{x}_{n}$ and $\theta_{n}$;

2) the standard UKF using equations (33) with state variables $\mathbf{x}_{n}$ and $\theta_{n}$

3) the recent Invariant-EKF (IEKF), an EKF variant on Lie groups that comes with guaranteed convergence properties, see [28] for its application to system (33)-(34), and with consistency properties [10];

4) the proposed Left-UKF-LG of Section IV using the Lie group embedding (36) and state variable $\chi_{n}$. 


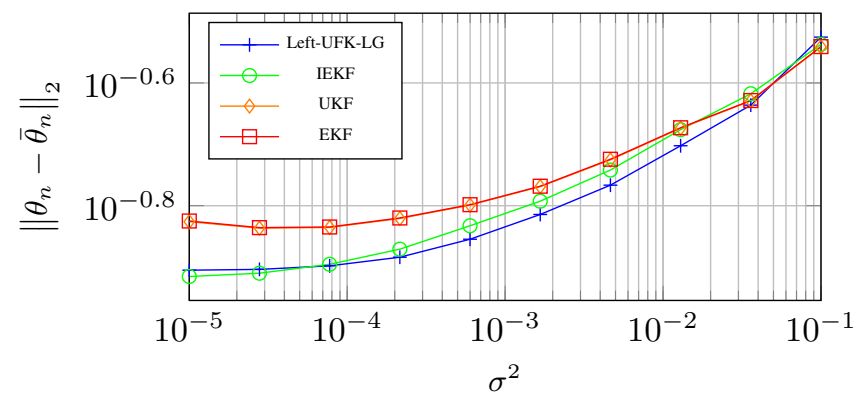

Fig. 3: Monte-Carlo average of the Root Mean Square Error on $\left(\theta_{n}\right)_{1 \leq n \leq N}$ over the whole trajectory, as a function of the noise measurement variance $\sigma^{2}$. Standard UKF and EKF achieve identical RMSE.

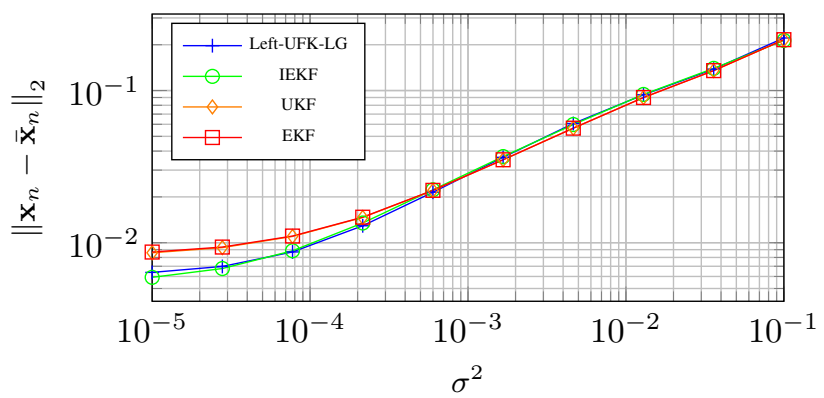

Fig. 4: Monte-Carlo average of the Root Mean Square Error on $\left(\mathbf{x}_{n}\right)_{1<n<N}$ over the whole trajectory, as a function of the noise measurement variance $\sigma^{2}$. Standard EKF and UKF achieve identical RMSE.

\section{B. Results}

To compare the proposed Left-UKF-LG ${ }^{2}$ with other methods for different levels of noise, we provide each filter with the raw odometer inputs $\boldsymbol{\omega}_{n}$, and we artificially add Gaussian noise to the ground truth position measurements to simulate noisy position measurements, delivered to the filter at a rate of $1 \mathrm{~Hz}$. Initially, we set for the filters both incorrect heading $\bar{\theta}_{0} \sim \mathcal{N}\left(0,(\pi / 2)^{2}\right)$ and position $\bar{x}_{0}^{1} \sim \mathcal{N}(0,1 / 8), \bar{x}_{0}^{2} \sim$ $\mathcal{N}(0,1 / 8)$. We then run 500 Monte-Carlo simulations for different levels of measurement noise $\sigma^{2}=\left[10^{-5}, 10^{-1}\right] \mathrm{m}^{2}$ and compare the (average) Root Mean Square Errors w.r.t. the ground truth over the whole trajectory. Results are displayed in figures Fig. 3 and Fig. 4.

\section{Discussion}

For this trajectory, Left-UKF-LG and IEKF provide best position estimates at low noise. Regarding the heading, IEKF is slightly better than Left-UKF-LG at low noise but Left-UKFLG becomes the most efficient filter when noise is moderate. We thus see that the Left-UKF-LG takes advantage of both the geometry-based structure of the IEKF, and the robustness of the unscented method. The following points seem important to us:

- The problem at hand (33) can be treated without the Lie group machinery, using $\mathbf{x}_{n}$ and $\theta_{n}$ as vector state

\footnotetext{
${ }^{2}$ We similarly implemented the Right-UKF-LG but we show only LeftUKF-LG which is the most efficient for the measurement (34).
}

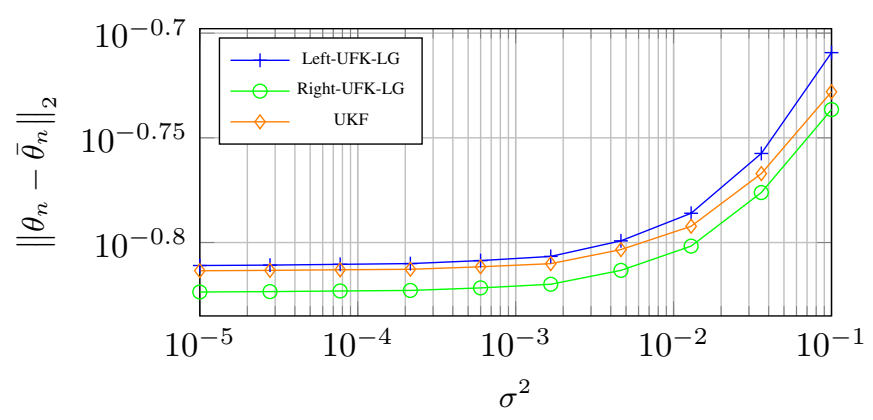

Fig. 5: Root Mean Square Error on $\left(\theta_{n}\right)_{1 \leq n \leq N}$ as a function of the noise measurement variance $\sigma^{2}$. We clearly remark the superiority of the Right-UFK-LG compared to both Left-UFK-LG and UKF.

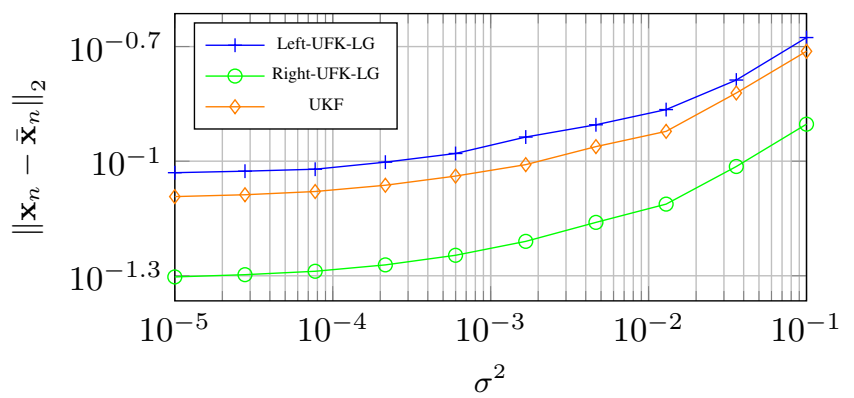

Fig. 6: Root Mean Square Error on $\left(\mathbf{x}_{n}\right)_{1 \leq n \leq N}$ as a function of the noise measurement variance $\sigma^{2}$. We clearly remark the superiority of the Right-UFK-LG compared to both Left-UFK-LG and UKF.

variables. However, we see that embedding it into a Lie group framework yields more powerful variants of the Kalman filter (the IEKF and the Left-UKF-LG).

- One could think the standard UKF systematically performs better than the standard EKF, especially when the measurement noise is high, due to its second order properties. This is generally true, but not always, as evidenced by the considered experiment.

- On the other hand, it seems the Lie group counterpart of the UKF improves the Lie group counterpart of the EKF, that is, the IEKF.

\section{Differences Between Left-UKF-LG and Right-UKF-LF}

In the latter section, we focused on the comparison between Left-UKF-LG and some existing filters. We illustrate in this section that there may be discrepancies between the performances of Left-UKF-LG and Right-UKF-LF. To do so we still consider the system model (36) but with alternative measurements

$$
\begin{aligned}
& \mathbf{Y}_{n}^{j}=\mathbf{R}\left(\theta_{n}\right)\left(\mathbf{x}_{n}-\mathbf{p}^{j}\right)+\mathbf{v}_{n}^{j}, j=1, \ldots, J, \text { where } \\
& \mathbf{R}\left(\theta_{n}\right)=\left[\begin{array}{cc}
\cos \left(\theta_{n}\right) & -\sin \left(\theta_{n}\right) \\
\sin \left(\theta_{n}\right) & \cos \left(\theta_{n}\right)
\end{array}\right],
\end{aligned}
$$

which represent a range-and-bearing observation of a sequence of known features located at $\mathbf{p}^{j} \in \mathbb{R}^{2}$ for $j=1, \ldots, J$, and where we let $\mathbf{R}(\theta)$ be the planar rotation of angle $\theta$. In our setting, we define $J=3$ features $\mathbf{p}^{1}=[12]^{\top}$, $\mathbf{p}^{2}=[-0.50]^{\top}$ and $\mathbf{p}^{3}=[01]^{\top}$. We then run 200 MonteCarlo simulations for different levels of measurement noise 
$\sigma^{2}=\left[10^{-5}, 10^{-1}\right] \mathrm{m}^{2}$ and compared the Root Mean Square Error w.r.t. the ground truth. Results are displayed in figures Fig. 5 and Fig. 6. They clearly reveal that the Right-UKF-LG outperforms both Left-UKF-LG and UKF in this particular case. In the absence of further insight, we thus recommend to the practitioner to design, implement and compare both UKF$\mathrm{LG}$ variants, and then select the most efficient of them.

\section{CONCLUSION}

We presented in this paper different solutions to Bayesian estimation problems on matrix Lie groups based on the UKF methodology, when the measurements consist of noisy partial measurements of the state, or full state noisy measurements of the state. We then applied these estimators to derive an UKF on Lie groups that comes in two variants. Experimental data combined with extensive Monte-Carlo simulations at various measurement noise levels illustrated the systematic superiority of the approach over the standard UKF on a robot localization example. Future work involves applications to drone navigation and to SLAM.

\section{ACKNOWLEDGMENT}

The authors would like to thank Martin BARCZYK, Tony NoËL for their precious help with the experiments.

\section{APPENDIX A}

\section{SPECIAL EUCLIDEAN 2D GRouP}

The Special Euclidean 2D $S E(2)$ group represents rigid transformations in 2D space and has $q=3$ parameters: heading $\theta$ and position $\mathbf{x}=\left[x^{1} x^{2}\right]^{\top}$. The elements $\chi \in S E(2), \boldsymbol{\xi}^{\wedge} \in$ $\mathfrak{g}$, are given, respectively, as

$$
\chi=\left[\begin{array}{ccc}
\cos (\theta) & -\sin (\theta) & x^{1} \\
\sin (\theta) & \cos (\theta) & x^{2} \\
0 & 0 & 1
\end{array}\right] \text { and } \boldsymbol{\xi}^{\wedge}=\left[\begin{array}{ccc}
0 & -\xi^{1} & \xi^{2} \\
\xi^{1} & 0 & \xi^{3} \\
0 & 0 & 0
\end{array}\right]
$$

The exponential and logarithm maps are obtained as

$$
\exp (\boldsymbol{\xi})=\left[\begin{array}{cc}
\mathbf{R}\left(\xi^{1}\right) & \mathbf{V}_{\boldsymbol{\xi}}\left[\begin{array}{l}
\xi^{2} \\
\xi^{3}
\end{array}\right] \\
\mathbf{0} & 1
\end{array}\right], \log (\boldsymbol{\chi})=\left[\begin{array}{c}
\mathbf{V}_{\underset{\theta}{\chi} \mathbf{x}}^{-1} \\
\dot{\theta}
\end{array}\right]
$$

where $\mathbf{R}\left(\xi^{1}\right)$ is defined in (39),

$$
\begin{aligned}
\mathbf{V}_{\boldsymbol{\xi}} & =\frac{1}{\xi^{1}}\left[\begin{array}{cr}
\sin \left(\xi^{1}\right) & -1+\cos \left(\xi^{1}\right) \\
1-\cos \left(\xi^{1}\right) & \sin \left(\xi^{1}\right)
\end{array}\right] \text { and } \\
\mathbf{V}_{\boldsymbol{\chi}} & =\frac{1}{\theta}\left[\begin{array}{cc}
\sin (\theta) & -1+\cos (\theta) \\
1-\cos (\theta) & \sin (\theta)
\end{array}\right],
\end{aligned}
$$

and see [29] for adjoint.

\section{REFERENCES}

[1] S. Julier and J. Uhlmann, "A New Extension of the Kalman Filter to Nonlinear Systems," in AeroSense'97, pp. 182-193, 1997.

[2] S. Julier and J. Uhlmann, "Unscented Filtering and Nonlinear Estimation," Proceedings of the IEEE, vol. 92, no. 3, pp. 401-422, 2004

[3] G. S. Chirikjian, Stochastic Models, Information Theory, and Lie Groups, Volume 2: Analytic Methods and Modern Applications. Springer Science \& Business Media, 2011.

[4] G. S. Chirikjian and M. Kobilarov, "Gaussian Approximation of Nonlinear Measurement Models on Lie Groups," in Decision and Control (CDC), pp. 6401-6406, IEEE, 2014.
[5] T. D. Barfoot and P. T. Furgale, "Associating Uncertainty with ThreeDimensional Poses for Use in Estimation Problems," IEEE Transactions on Robotics, vol. 30, no. 3, pp. 679-693, 2014.

[6] A. Barrau and S. Bonnabel, "Intrinsic Filtering on Lie Groups with Applications to Attitude Estimation," IEEE Transactions on Automatic Control, vol. 60, no. 2, pp. 436-449, 2015.

[7] K. Wolfe, M. Mashner, and G. S. Chirikjian, "Bayesian Fusion on Lie groups," Journal of Algebraic Statistics, vol. 2, no. 1, pp. 75-97, 2011.

[8] S. Diemer and S. Bonnabel "An Invariant Linear Quadratic Gaussian Controller for a Simplified Car," in Robotics and Automation (ICRA), 2015 IEEE International Conference on, pp. 448-453, IEEE, 2015.

[9] C. Hertzberg, R. Wagner, U. Frese, and L. Schröder, "Integrating Generic Sensor Fusion Algorithms with Sound State Representations Through Rncapsulation of Manifolds," Information Fusion, vol. 14, no. 1, pp. 57-77, 2013.

[10] A. Barrau and S. Bonnabel, "An EKF-SLAM Algorithm with Consistency Properties," arXiv preprint arXiv:1510.06263, 2015.

[11] R. V. der Merwe and E. A. Wan, "The square-root unscented kalman filter for state and parameter-estimation," in IEEE International Conference on Acoustics, Speech, and Signal Processing. Proceedings, vol. 6, pp. 3461-3464, 2001.

[12] J. L. Crassidis and F. L. Markley, "Unscented Filtering for Spacecraft Attitude Estimation," Journal of guidance, control, and dynamics, vol. 26, no. 4, pp. 536-542, 2003.

[13] T. Lee, "Global Unscented Attitude Estimation via the Matrix Fisher Distributions on SO(3)," in American Control Conference (ACC), 2016, pp. 4942-4947, IEEE, 2016.

[14] J. Bohn and A. K. Sanyal, "Unscented State Estimation for Rigid Body Motion on SE(3)," in Decision and Control (CDC), 2012 IEEE 51st Annual Conference on, pp. 7498-7503, IEEE, 2012.

[15] J. J. Bohn, A. K. Sanyal, and E. A. Butcher, "Unscented State Estimation for Rigid Body Attitude Motion with a Finite-time Stable Observer," in Decision and Control (CDC), 2016 IEEE 55th Conference on, pp. 4698-4703, IEEE, 2016.

[16] J.-P. Condomines, C. Seren, and G. Hattenberger, "Nonlinear State Estimation Using an Invariant Unscented Kalman Filter," in AIAA GNC 2013, AIAA Guidance, Navigation and Control Conference, (Boston, United States), pp. 1-15, AIAA, 2013.

[17] J.-P. Condomines, C. Seren, and G. Hattenberger, "Pi-Invariant Unscented Kalman Filter for Sensor Fusion," in Decision and Control (CDC), pp. 1035-1040, IEEE, 2014.

[18] Y. Wang and G. S. Chirikjian, "Error Propagation on the Euclidean Group with Applications to Manipulator Kinematics," IEEE Transactions on Robotics, vol. 22, no. 4, pp. 591-602, 2006.

[19] A. W. Long, K. C. Wolfe, M. Mashner, and G. S. Chirikjian, "The Banana Distribution is Gaussian: A Localization Study with Exponential Coordinates," in Robotics: Science and Systems VIII, 2012.

[20] G. Bourmaud, R. Mégret, M. Arnaudon, and A. Giremus, "ContinuousDiscrete Extended Kalman Filter on Matrix Lie Groups Using Concentrated Gaussian Distributions," Journal of Mathematical Imaging and Vision, vol. 51, no. 1, pp. 209-228, 2015.

[21] A. Barrau and S. Bonnabel, "Intrinsic Filtering on $\mathrm{SO}(3)$ with Discretetime Observations," in Decision and Control (CDC), 2013 IEEE 52nd Annual Conference on, pp. 3255-3260, IEEE, 2013.

[22] G. Loianno, M. Watterson, and V. Kumar, "Visual Inertial Odometry for Quadrotors on SE(3)," in 2016 IEEE International Conference on Robotics and Automation (ICRA), pp. 1544-1551, 2016.

[23] S. Hauberg, F. Lauze, and K. S. Pedersen, "Unscented Kalman Filtering on Riemannian Manifolds," Journal of mathematical imaging and vision, vol. 46, no. 1, pp. 103-120, 2013.

[24] G. S. Chirikjian, Stochastic Models, Information Theory, and Lie Groups, Volume 1: Classical Results and Geometric Methods. Applied and numerical harmonic analysis, Birkhäuser, 2009.

[25] J. Dunik, M. Simandl, and O. Straka, "Unscented Kalman Filter: Aspects and Adaptive Setting of Scaling Parameter," IEEE Transactions on Automatic Control, vol. 57, 2012.

[26] T. Hervier, S. Bonnabel, and F. Goulette, "Accurate 3D maps from depth images and motion sensors via nonlinear Kalman filtering," in Intelligent Robots and Systems (IROS), 2012 IEEE/RSJ International Conference on, pp. 5291-5297, IEEE, 2012.

[27] A. De Luca, G. Oriolo, and C. Samson, Feedback Control of a Nonholonomic Car-like Robot, pp. 171-253. Springer, 1998.

[28] A. Barrau and S. Bonnabel, "The Invariant Extended Kalman Filter as a Stable Observer," IEEE Transactions on Automatic Control, vol. 62, no. 4, pp. 436-449, 2017.

[29] E. Eade, "Lie Groups for 2D and 3D Transformations," 2013. 